\title{
Deep Learning towards Autonomous Ship Navigation and Possible COLREGs Failures
}

\author{
Lokukaluge P. Perera \\ UiT The Arctic University of Norway \\ Tromso, Norway \\ Prasad.Perera@uit.no
}

A structured technology framework to address navigation considerations, including collision avoidance, of autonomous ships is the focus of this study. That consists of adequate maritime technologies to achieve the required level of navigation integrity in ocean autonomy. Since decision-making facilities in future autonomous vessels can play an important role under ocean autonomy, these technologies should consist of adequate system intelligence. Such system intelligence should consider localized decision-making modules to facilitate a distributed intelligence type strategy that supports distinct navigation situations in future vessels as agent-based systems. The main core of this agent consists of deep learning type technology that has presented promising results in other transportation systems, i.e. self-driving cars. Deep learning can capture helmsman behavior, therefore such system intelligence can be used to navigate future autonomous vessels. Furthermore, an additional decision support layer should also be developed to facilitate deep learning type technologies, where adequate solutions to distinct navigation situations can be facilitated. Collision avoidance under situation awareness, as one of such distinct navigation situations (i.e. a module of the decision support layer), is extensively discussed. Ship collision avoidance is regulated by the COLREGs under open sea areas. Hence, a general overview of the COLREGs and its implementation challenges, i.e. possible regulatory failures, under situation awareness of autonomous ships is also presented with the possible solutions. Additional considerations, i.e. performance standards with the applicable limits of liability, terms, expectations and conditions, towards evaluating ship behavior as an agent-based system in collision avoidance situations are also illustrated.

Keywords: Autonomous ship, ship intelligence, deep learning, agent-based systems, COLREGs, situation awareness, collision avoidance.

\section{INTRODUCTION}




\section{Autonomy in the Ocean}

Autonomy that can be expressed as the right or condition of freedom from external controls or influences is an important quality, i.e. an entity can have. However, the same entity should have adequate system intelligence to make appropriate decisions in relation to possible internal and external variations. Therefore, the existence of the same entity can be preserved, i.e. by system intelligence. This concept can be adopted towards mechanical and electrical systems by introducing machine intelligence-based decision-making facilities to operate themselves. Such systems are often categorized as intelligent machines and these self-operating systems, i.e. autonomous systems, should consist of adequate decision support tools and techniques. However, various advanced technologies to support and evaluate such intelligent machines are yet to be matured under the respective industries. Several such examples can be found in the recent technological advancements of autonomous systems, i.e. self-driving cars, robots, etc. [1] and that have made the initial pathway towards the development of advanced decision-making facilities. Furthermore, such decision-making facilities have also been supported by internal and external loT (i.e. internet of things), big data and communication infrastructure to overcome the respective challenges in autonomous systems. The success of decision-making facilities in such autonomous systems should be evaluated, comprehensively and their decision integrity should be quantified by the respective authorities and future researchers [2]. 
Autonomous systems will play an important role in the future transportation systems, even though there are many technological challenges that should be addressed by the respective industries [3]. Since self-driving vehicles have already been introduced on public roads, it is expected that similar systems will also be introduced by maritime and air transportation in the near future. On the other hand, there are several initiatives to introduce such technologies in experimental scales into these transportation systems have also been started [4]. Hence, this study focusses to address the required navigation technologies, i.e. including collision avoidance, of maritime transport systems with autonomous and remote-controlled capabilities. Since future vessels need the same technologies as a part of ocean autonomy, i.e. where autonomous, manned and remote-controlled ships are interacting with various distinct navigation situations, that should consist of adequate system intelligence to facilitate such situations. Therefore, this study introduces a structured technology framework, i.e. a ship intelligence framework, that consists of various modules to support distinct navigation situations of autonomous and remote-controlled ships.

Modern vessels are facilitated with onboard and onshore IoT to support various digitalization applications of the shipping industry. Industrial digitalization converts conventional paper-based information handling approaches into data driven applications. A detailed overview of vessel and ship system behavior can be captured under industrial digitalization and that information should be used to enhance decisionmaking facilities of future ships. Hence, industrial digitalization should support ocean autonomy, where human inference can be minimal by transforming big data collected 
by onboard and onshore loT into information and then that information into intelligent operational and navigational decisions of future vessels [4].

There are several millstones that should be achieved by the shipping industry in order to make autonomous shipping a reality. "Remote Controlled Ship" can be an important milestone in this route [5] and this same state, i.e. remote controlled navigation, can always be a part of autonomous ship navigation. In general, each voyage can have both autonomous and remote controlled navigation sectors that should be segmented by considering vessel operational requirements. Such operational requirements may also be influenced by the collision risk among various ship encounters. e.g. high collision risk areas can be navigated under the remote controlled mode.

The required maritime infrastructure to support both remote controlled and autonomous ship operations should be developed and that can also be another important milestone in the same route [6]. Furthermore, the success in ship intelligence, i.e. artificial intelligence (AI), to navigate and operate vessels and ship systems, can make the next important milestone in this route. Various machine-learning algorithms, as a part of Al can support to achieve the required integrity levels of ship intelligence, should be included in this step. Appropriate decision support facilities to enhance ship intelligence should also be considered and that will be another important milestone in this route. Finally, adequate tools and techniques to evaluate vessel behavior, i.e. as a part of ship intelligence, with decision support facilities should also be developed, 
where the acceptable risk and limits of liability, terms, expectations and conditions under autonomous ship navigation should be defined, adequately.

\section{Agent Based Systems}

It is expected that autonomous ships will be agent based systems and the same concept can also satisfy the respective milestones, appropriately. An agent can be defined as a system that is located in a specific environment interacts with the same environment by intelligent decisions and actions to satisfy its design objectives. However, other similar and/or different agents can also be located within the same environment, where these agents may interact. Therefore, various cooperative and non-cooperative interactions among the agents should also be expected in such an environment. Since each agent may have its own design objectives, adequate system intelligent to fully or partially satisfy the same in the respective environment should be facilitated. If such individual design objectives cannot be achieved (i.e. unsatisfactory) in this environment, adequate compromising strategies to satisfy appropriate group objectives should be considered. Such situations can be categorized as a cooperative multi-agent learning approach, where additional machine learning approaches (i.e. reinforcement learning) [7] can be incorporated. Therefore, adequate system intelligence in these agents should be included to handle rather complex interactions among themselves and satisfy their design objectives in complex environmental conditions.

Since autonomous ships will represent agent based systems, various cooperative and non-cooperative interactions among the respective vessels in open sea areas and 
traffic lanes are expected in the future. In general, such an intelligent agent should consist of the following basic properties [8]:

- Autonomy: Each agent should operate by its own decisions/actions and/or internal states without any direct inference from humans or others.

- Social-ability: Each agent should interact with other agents (i.e. including humans) by an appropriate agent-communication language.

- Reactivity: Each agent should not only interact with its environment but also respond to the respective environmental changes and challenges in a timely fashion.

- Pro-activeness: Each agent should take appropriate initiatives to exhibit goaloriented behavior, i.e. to satisfy its design objectives, in the respective environment.

One should note that these properties should also be a part of future autonomous vessels as agent based systems, therefore that can be used to support the respective interactions among the vessels in an ocean environmental. However, these vessels should have adequate ship intelligence with decision support facilities to enhance these agent-based properties and that can overcome the respective challenges in autonomous ship navigation in a mixed environment, where remote-controlled, autonomous and manned vessels are interacting.

\section{AUTONOMOUS VESSELS AND SHIP SYSTEMS}

Future Vessels and Onboard Systems 
Remote-controlled vessels that will open the path towards autonomous vessels create the next generation maritime transport systems. Human presence will be limited in fully autonomous vessels, therefore ship systems that support humans onboard, i.e. accommodation systems, air-conditioning, etc., can be eliminated. Hence, the costs of installing human support systems and their maintenance can also be eliminated. Since remote-controlled and autonomous vessels may not require to have the comfort levels that are designed for humans, the shapes and sizes of such vessels can be designed in a way that reduce the respective ship resistance, further. That can be done in several levels. The reduction in deck and super-structure, i.e. not required to support human presence, in such vessels can reduce the respective air resistance. Since the comfort of humans may not be an issue in these vessels, more slender hulls with low clam water ship resistance can be considered. The space for humans and human support systems can be eliminated, therefore the vessel size can be reduced, i.e. vessel underwater volume can be reduced and that reduces clam water ship resistance. On the other hand, the cargo carried by such vessels can be increased with the same vessel size and that can increase the respective energy efficiency of future vessels, significantly. Hence, it is expected that the shapes and sizes of future vessels will be changed to satisfy remotecontrolled and autonomous operational conditions. That will also reduce the respective operational costs and increase ship energy efficiency. However, additional vessel stability and maneuverability issues that may rise due such ship designs should also be addressed in the future. 
On the other hand, new ship systems, i.e. ship intelligence with decision support facilities, to enhance remote-controlled and autonomous operations will be installed in these vessels. That can introduce new system installation and maintenance costs into these ships. It is expected that the reliability of these ship systems should considerably be higher to cope with harsh environmental conditions and unexpected system failures. Otherwise, that can lead towards possible ship collisions and environmental disasters. Hence, it is a requirement that future vessels and ship systems should continuously be supported by onboard and onshore condition monitoring and condition-based maintenance applications to preserve their availability.

Onboard and onshore loT collect ship performance and navigation data sets in real-time. Such network connectivity in these vessels will create big data sets, where the respective information of vessel navigation and ship system operations can be extracted. The same information can be used to support various vessel related applications. e.g. the ship system health condition possibly at a component level. The respective system and component failures can often be identified at an early stage due to their health information and that should be an important outcome of ship performance and navigation data sets. Since the reliability of ship systems can play a crucial role in autonomous and remote controlled vessels, various recent research and development activities are focused on developing conditions monitoring (CM) and condition based maintenance (CBM) applications. There are several onshore condition monitoring centers, i.e. intelligent asset management centers to utilize ship 
performance and navigation data sets, are also introduced to support future vessels by several maritime related service companies [9].

\section{Ship Intelligence}

Since this study focusses to address the required navigation technologies to support future vessels, a structured framework with various technology components to support ship navigation is discussed in this section. It is expected that future autonomous vessels will be trained by humans through ship navigation data sets, i.e. to navigate like an experienced helmsman. Similar training approaches have successfully been implemented by other transport systems, i.e. autonomous car, and drones [10], that can be adopted towards the maritime transport systems. This approach can be seen as cloning human behavior through smart systems. However, adequate intelligence should be developed within these systems to absorb such knowledge from human navigators during the training processes. The same system intelligence, i.e. so called ship intelligence, should be able to transfer from one vessel to another among sister vessels and that can further be improved by navigation data sets collected by additional ship maneuvers. In general, ship intelligence in autonomous vessels should consist of complex system architecture supported by a decision support layer to facilitate adequate solutions to distinct navigation situations. The proposed structured technology framework of ship intelligence, to support autonomous ship navigation, with the required systems (i.e. navigation and automation systems) is presented in Figure 1. 
Initially, ship navigators will be able to train autonomous vessels to achieve the respective system intelligence levels, i.e. to operate both navigation and automation systems. The training process should be done by humans, i.e. onboard vessels and onshore remote-controlled centers, where navigation decisions (human inference) with respect to various path following type ship maneuvers can be executed. Those decisions should be converted into navigation actions, i.e. propeller/thruster and rudder control actions. The navigation actions that need to satisfy the required ship route and motions vs actual ship route and motions can be considered as the first level inputs to the training process (i.e. navigation training) of ship intelligence (see Figure 1). The actual ship route and motions are represented by the advanced ship predictor (ASP), i.e. an algorithm that estimates the present and future vessel positions and orientations. The required ship route and motions are represented by the digital ship route (DSR), i.e. an algorithm that visualizes the navigation path that the vessel should take. Hence, the ship navigator should take appropriate decisions by considering the ASP and DSR and execute on the ship automation system, i.e. propeller/thruster and rudder control systems, to achieve the required vessel behavior i.e. ship speed, heading and course.

One should note that these propeller/thruster and rudder control system configurations can vary from one vessel to another. Therefore, the navigators' actions on propeller (i.e. propeller pitch and speed) and rudder (i.e. rudder angle) control systems can also vary due to the same reasons. Even though that may effect on the training process of autonomous vessels, that can be similar among sister vessels. 
Furthermore, vessel seakeeping and maneuvering behavior can be influenced by vessel structures, ship systems and external environmental conditions, other than the navigators' actions. Both vessel behavior and external environmental conditions should be observed by onboard and onshore loT through vessel navigation information. Each voyage consists of the required ship route and motions (i.e. through the DSR), therefore appropriate navigation actions should be taken by the helmsman to achieve the same with respect to the actual ship route and motions (i.e. through the ASP), as mentioned before.

Ship navigation systems can consist of the ECDIS (Electronic chart display and information system), Radar and APRA (Automatic radar plotting aid), Conning and additional loT. These additional loT of future vessels can include the GNSS (Global navigation satellite system), INS (Inertial navigation system), LiDAR (Light detection and ranging), digital cameras and weather transmitters. A combination of such systems can be classified as an integrated bridge system (IBS) [11] in the future. IBSs can collect and visualize ship navigation and automation information, including vessel seakeeping and maneuvering behavior, in an actual ship route (i.e. vessel position, speed, course, heading, draft, etc.). This information is collected as big data sets that should further be analyzed to extract the respective ship navigation and automation information, including vessel seakeeping and maneuvering behavior. The accumulation of seakeeping and maneuvering behavior and external environmental conditions collected by loT along with the navigator's actions are considered as the second level inputs to the training process of ship intelligence. These two inputs, i.e. navigator's actions and 
vessel navigation and automation information including seakeeping and maneuvering behavior under the respective external environmental conditions, complete the typical training cycle, i.e. Training Process in Figure 1, of autonomous vessels. However, there are additional layers that should facilitate ship intelligence of autonomous vessels to maneuver in distinct situations, where additional navigational challenges can be observed. These system layers are further illustrated in Figure 1 as decision support, information sources and supporting services and authorities.

\section{Deep Learning in Shipping}

Ship intelligence consists of a deep learning based mathematical framework, i.e. an artificial neural network. The same framework can create the respective agent behavior within autonomous vessels. Similar frameworks have been implemented by other transport systems, i.e. autonomous navigation systems of drive-less cars, and achieved promising results in terms of navigating within the required safety levels [12]. In general, a deep leaning based mathematical framework, e.g. convolutional neural networks (CNNs, or ConvNets), transform a self-driving vessel problem into a data classification problem. CNNs are a class of deep neural networks [13] that can handle complex image classification problems and the outcome can be used as system intelligence for self-driving vehicles.

The same image classification approach can provide an elegant mechanism to capture helmsman behavior, i.e. agent behavior in ship navigation. At an initial stage, the CNNs should be the observers for manual or remote controlled vessels that are operated by human navigators. That step is previously categorized as the Training 
Process (see Figure 1) and the main objective of this phase is to train the respective neural networks to capture ship behavior with respect to navigator's actions. It is expected that adequate information on vessel seakeeping and maneuvering behavior can also be accommodated into these neural networks to enhance self-driving capabilities of future autonomous ships.

When these neural networks are adequately trained, that technology can be used to navigate the first generation autonomous vessels. In addition, the same deep learning based mathematical frameworks can be distributed (i.e. shared knowledge) among sister vessels and that can also be further trained under their operational conditions. One should note that these networks should be supported by image based information and navigator's actions rather than system parameters during the training phase.

These networks, i.e. the behavioral knowledge, can be transferred from one vessel to another among sister vessels as mentioned before, however additional training may require to further improve the respective ship intelligence in some situations. If these vessels are standardized during the ship design phase, then such additional training requirements can be eliminated. After a successful navigation training period, ship intelligence should be able to navigate the respective vessel as an experienced helmsman, i.e. that is represented by the execution process in Figure 1 . Several technological challenges in navigating ocean-going vessels, specially under rough weather conditions, can also be expected. Conventional vessels are often categorized as under-actuated systems, i.e. rudder and propeller/thruster control systems may not 
able to control vessels completely during the phase of ship navigation. This ship controllability issue, i.e. under-actuation, can complicate the training process of autonomous ships. One should note that drones or under water vehicles navigate in a single environmental media (i.e. land, air or water). On the other hand, ships are navigating between two environmental media (i.e. air and water) and the respective interactions between both media (i.e. wind and wave conditions) can introduce complex seakeeping and maneuvering behavior into these vessels. That can complicate the training process of ship intelligence, i.e. due to ship under-actuation. Furthermore, the heavy inertia in ocean going vessels can introduce additional complexity into ship underactuation, especially under rough weather conditions.

The rudder and propeller control systems, i.e. only available control units for vessel actuation, may fail to control such vessels under rough weather conditions. While ships are navigating in moderate or high speed (i.e. over 3-4 knots) conditions, the capabilities of thrusters are negligible. Furthermore, the control solutions developed for autonomous surface vehicles (ASVs) and autonomous underwater vehicles (AUVs) may not be acceptable for large vessels due to the same reasons. Similarly, the control solutions developed for autonomous land vehicles, i.e. driver-less car, may not be acceptable due to the same reasons, i.e. land vehicles are light weight transportation units compared to ships and have the better controllability over the roads. Hence, the controllability of under-actuated vessels in complex navigation conditions can be a major challenge in autonomous ocean navigation. However, it is expected that deep 
learning based mathematical frameworks supported by the decision support layer (see Figure 1) may overcome such challenges in ship navigation, as discussed before.

\section{Decision Support Facilities}

The decision support layer with various onboard and onshore loT should support ship intelligence to navigate future vessels (see Figure 1). Each ship route can be divided into several voyage segments during the planning phase and that can be classified into possible autonomous and remote-controlled navigation segments. Some voyage segments of ship navigation may execute as remote-controlled routes due to the respective safety reasons. Global and local digital maps including navigation and emission control rules and regulations should also support the same voyage planning phase as presented in Figure 1. Furthermore, additional decision support facilities such as weather routing and pilotage can also be a part of voyage planning. Even though global maps are already included under the ECDIS, the local maps (i.e. harbor areas and confined waters) with the navigation rules and regulations can be supplied by the respective maritime and port authorities during the ship operation phase to enhance vessel autonomous capabilities.

Such local information can improve the safety of autonomous ship navigation, therefore the pilotage type activities, i.e. deploy humans with local navigation knowledge to guide vessels, can be eliminated. In addition, the respective maritime authorities can enforce the environmental regulations, i.e. energy efficiency and emission control rules and regulations [14], on these vessels by distributing the respective information through the decision support layer. These energy efficiency and 
emission control rules and regulations are enforced, extensively on the designated emission control areas (ECAs). Therefore, the local digital maps can provide such information, accurately to enforce the respective rules and regulations on ship navigation.

Local digital maps can be integrated with global maps to support autonomous ship navigation under SLAM (Simultaneous localization and mapping) type applications [15]. SLAM type applications can support intelligent agents, i.e. autonomous vessels, to locate themselves within integrated local maps in a global scale by the information collected by onboard and onshore loT. These agents can also learn the same digital environment by executing possible actions during the phase of ship navigation. Furthermore, additional sensors, i.e. Lidar and Laser, can also be available in autonomous vessels to enhance SLAM capabilities. Additional information resources such as VTI (vessel traffic information), TSS (Traffic separation Schemes), AIS (Automatic identification system) and LRIT (Long-Range Identification and Tracking) can also support the same applications. These information sources should be provided and regulated by vessel traffic management and information systems (i.e. VTMIS) to improve the navigation safety of future vessels.

Weather routing and safe ship handling type applications should also function under the same decision support layer to improve the safety and efficiency of vessel navigation (see Figure 1). The required global and local weather information can be obtained from various weather centers/sources by autonomous vessels. Weather routing can facilitate by providing the recommended ship routes to ocean going vessels 
prior to and during each voyage under the respective navigational constraints and global weather forecasts [14]. Safe ship handling can facilitate by providing appropriate ship position, orientation and speed conditions on the recommended route under the same conditions [16] of ocean going vessels, especially in unexpected rough weather conditions. Therefore, both weather routing and safe ship handling should complement each other under global and local weather forecasts to support the voyage planning phase. Furthermore, weather routing and safe ship handling should also complement each other during the operation phase of ship intelligence to achieve the required safety levels of autonomous ship navigation.

Conventional ships consist of stability calculation systems to estimate the vessel loading conditions. Due to the cargo loading and unloading activities at ports, the stability condition of each vessel should be calculated the beginning of its voyage. It is expected that autonomous vessels will have similar decision support facilities with additional loT to verify the respective weight/cargo distributions, accurately. Since future cargo loading and unloading conditions in posts will also be automated, that information can be shared with these decisions supporting layers of autonomous vessels.

Collision avoidance, under situation awareness, among stationary and moving objects can play an important role in autonomous ships in relation to the navigation safety. The stationary objects, i.e. land masks, shipwrecks, etc., are marked in global and local digital maps and unexpected ones should be detected by onboard loT. The collision avoidance of stationary objects may relate to path planning type 
approaches, especially in harbor or confined water navigation. Therefore, the decision support layer should consist of adequate sensors and algorithms to detect and identify stationary and moving objects. Such stationary and moving objects detection and classification applications have also been implemented under deep learning type frameworks with successful results [17], therefore that technology can be adopted towards autonomous ship navigation. This is another example, where the deep learning based mathematical frameworks can be implemented in autonomous vessels and that can also be a part of ship intelligence. The collision avoidance of moving objects, especially with ocean going vessels, in relation to the respective navigation rules and regulations is discussed in the following sections.

\section{Collision Avoidance under Situation Awareness}

Future vessels should be facilitated with appropriate collision avoidance technologies to improve the navigation safety. However, the behavior of such technologies should also be evaluated, therefore the respective system reliability levels in autonomous ship navigation $[18,19]$ can be preserved. This section illustrates the respective procedure and challenges in evaluating the collision avoidance technologies under situation awareness of autonomous ship navigation. In general, the evaluation procedure for autonomous ship navigation should consist of the following basic units:

- A legal framework and its possible regulatory failures.

- Autonomous and target vessels and their behavior.

- A testing system to evaluate vessel behavior.

Legal Framework and its Possible Regulatory Failures 
All ocean going vessels should follow the law of the sea. The International Maritime Organization (IMO) in 1972 by the Convention on the International Regulations for Preventing Collisions at Sea (COLREGs) has introduced a legal framework to regulate ship encounter situations [20]. The respective studies of ship collisions indicate that $75 \%-96 \%$ of maritime collisions and causalities caused by some types of human errors and $56 \%$ of major maritime collisions involved one or more violations of the COLREGs [21]. It is expected that future ships should also be regulated by the same rules and regulations during their encounter situations. Since collision avoidance decisions will be taken by ship intelligence facilitated by the decision support layer, the respective behavior of such vessels should be evaluated, i.e. by testing systems, under the acceptable performance standards. Three distinct ship encounter situations that have the risk of collisions can be considered by the testing system: overtaking, head-on, and crossing. One should note that all ship encounters can be classified into these navigation situations, where the respective collision avoidance decisions should be taken. In general, the decision space of ship collision avoidance in relation to a two vessel encounter situation can be categorized into the following stages in open sea under the COLREGs:

- When both vessels are beyond the general collision risk region (see Figure 2), both vessels have the options to take appropriate actions to avoid a possible collision situation.

- When both vessels are at the general collision risk region, the "give-way" vessel (i.e. the vessel has low priority for navigation) should take appropriate actions to 
achieve safe passing distance in accordance with the COLREGs, and the "stand on" vessel (i.e. the vessel has high priority for navigation) should maintain its course and speed.

- When both vessels are at the critical collision risk region, if the "give way" vessel does not take appropriate actions to achieve a safe passing distance in accordance with the COLREGs, then the "stand on" vessel has the option to take appropriate actions to avoid a possible collision situation.

- However, local navigation rules and regulations, traffic lanes, offshore operations and special types of vessels (i.e. fishing vessels) can override the same decisions, especially under overtaking and head-on situations.

It is observed that the COLREGs may have possible regulatory failure situations under the same decision space [22]. Such situations have been reported in [23], while applying the COLREGs into if-then-else type computer codes, i.e. under Fuzzy Logic. Those situations are further discussed in this section to illustrate possible COLREGs regulatory failure situations in autonomous vessels, where ship intelligence is making collision avoidance decisions.

Figure 2 represents a situation, where the target vessel locates in a head-on situation slightly to the port, with the own vessel. The respective own and target vessel positions $\left(O(k)\right.$ and $\left.P_{i}(k)\right)$, course-speed vectors $\left(V_{o}(k)\right.$ and $\left.V_{i}(k)\right)$ and relative navigational trajectory of the target vessel with respective the own vessel are also presented in the same figure. The respective head-on and crossing (i.e. from the starboard and port) regions are also denoted in the same figure. Therefore, the target 
vessel relative navigation trajectory is located in a fuzzy region between the head-on and crossing from port regions. The own and target vessel definitions are presented in with additional details in a ship encounter situations in [23]. This situation can create a regulatory failure situation within the COLREGs due to the fuzzy region and that is further explained in [24]. Hence, an ambiguity within the COLREGs can raise in this situation to define this either as a head-on or crossing situation and that may result in a possible collision situation.

Figure 3 represents a situation, where the target vessel relative navigational trajectory changes from a head-on situation to a crossing situation with respect to the own vessel. Therefore, such situations can also create a regulatory failure situation within the COLREGs, i.e. which rules either in head-on or crossing situations should be applied. Hence, an ambiguity within the COLREGs can also raise in this situation to define which rules should be applied. Figure 4 represents a situation, where multiple target vessels approach the own vessel from different directions. An ambiguity within the COLREGs can raise in this situation to define which vessel should be given the priority to avoid a possible collision situation. If the own vessel may fellow two contradictory rules accordance with the COLREGs in this situation, that may result in a possible collision situation.

One should note that these are possible regulatory failure situations that are observed in a simulated study [23], while applying to a COLREGs based decision support system in ship navigation. Therefore, additional regulatory failure situations are yet to be discovered in the future, when the COLREGs are implemented under the decision 
support layer (see Figure 1) of ship intelligence. Hence, adequate measures to overcome such regulatory failure situations within the COLREGs legal framework should be considered and that can enhance the required safety levels of autonomous ship navigation.

\section{Autonomous and Target Vessel Behavior}

Vessels may not honor rules and regulations in some navigation situations and that may create increased collision risk situations. One should note that such ship encounters are clear regulatory violations within the COLREGs legal framework and cannot be categorized as regulatory failure situations. That may result in possible ship collision and close encounter situations in open sea and the COLREGs legal framework may not provide clear guidance to overcome for such situations. However, it is the requirement that all vessels should avoid ship collision and close encounter situations by executing appropriate actions, accordance with the COLREGs. In general, ship navigators use their experiences to avoid such situations and that may also lead to "crash stopping" type maneuvers of the "stand on" vessel, i.e. due to a distance constraint for speed reduction.

The characteristics of the "stand on" vessel, i.e. its "stopping distance" and "turning circle", should be considered for executing appropriate collision avoidance actions in such situations. Since the expert knowledge can also be used to avoid close ship encounter situations, it is expected that the same knowledge can also be absorbed by a deep learning based mathematical framework during the training phase. Even though these increased collision risk situations can be unintentional, the respective 
decision support layer should support ship intelligence to execute appropriate collision avoidance actions in such situations. On the other hand, adequate tools and techniques to predict the intensions of encountering vessels should also be developed to support the same ship intelligence in autonomous vessels.

\section{Increased Collision Risk Ship Encounters}

In addition to possible regulatory failures, the system level responses (i.e. vessel behavior) of ship intelligence in autonomous vessels under collision risk encounters should also be evaluated and the acceptability of system level navigational actions should also be investigated. That can be done by having a testing system supported by appropriate performance standards to evaluate vessel behavior. These performance standards are likely to be defined by the respective maritime authorities or classification societies to support this process. However, the evaluation process of autonomous vessels under the critical collision risk region (see Figures 2, 3 and 4) should be carefully formulated, since the vessel behavior can also depend on vessel maneuverability characteristics. One should note that the COLREGs have not been provided any clear rules and regulations to avoid such situations. On the other hand, the maneuverability characteristics can be captured by ship intelligence under deep learning type mathematical frameworks. Therefore, ship intelligence may provide the initial guidelines of the respective performance standards to evaluate the first-generation autonomous vessels.

Distinct ship navigation situations with higher collision risk conditions should be recreated to evaluate vessel behavior, i.e. ship intelligence, under such testing 
platforms. The outcomes of vessel behavior under close ship encounter situations, should be communicated towards system developers of autonomous vessels to improve their ship intelligence and decision support facilities. Furthermore, the testing systems should have the capabilities to suggest the required autonomous ship behavior, i.e. to reduce the collision risk, and the appropriate reasons to illustrate such behavior under the respective legal frameworks, i.e. the COLREGs and local navigational rules and regulations. Any unusual vessel behavior or collision avoidance failures, i.e. regulatory failure situations, should initiate required modifications into the respective legal frameworks.

\section{Testing System for Vessel Behavior}

A general overview of a testing system with the required components to evaluate collision avoidance behavior of autonomous vessels is presented in Figure 5. That can be influenced by several legal frameworks i.e. the COLREGs and other local navigation rules and regulations. The systems should evaluate autonomous ship behavior as an agent based system under various vessel encounter situations, as mentioned before. Therefore, distinct navigation situations, i.e. various navigation routes with varying weather conditions, can also be introduced into the same to create realistic ocean going conditions. Autonomous vessel behavior can further be detailed into navigation and automation system levels by monitoring the respective systems. Therefore, the vessel and ship system behavior can be evaluated in a more detailed level by the testing system with the pre-defined performance standards. That can 
provide specific performance expectations that should be satisfied by autonomous vessels.

Appropriate course and/or speed changes, at any cost, must be taken by ocean going vessels to avoid possible collision situations, as highlighted in the COLREGs. Hence, that unique feature can introduce a minimum expectation level for the performance standards in the testing systems. However, the ultimate expectation of the performance standards may relate to selected policy options and/or legal frameworks introduced by the respective maritime authorities. Furthermore, that will also be influenced by the applicable limits of liability, terms, expectations and conditions introduced by the insurance industry. However, the existing performance standards [25, 26] for ship navigation can also be adopted by the same testing systems, initially.

The initial performance standards for autonomous vessels should be developed in relation to two-vessel encounter situations and that can be expanded towards multiple vessel encounter situations. Hence, the performance standards should consider the following navigation features to evaluate vessel behavior:

- Autonomous and target vessel domains.

- The collision risk between the vessels.

- The distance and time to a possible collision/close encounter situation.

- Autonomous and target vessels course-speed vectors.

- The bearing vector between two vessels.

- Autonomous vessel decisions and the time to execute the same decisions into actions. 
- Autonomous and target vessel predicted and actual behavior.

- The satisfactory level (i.e. under the performance standards) of the decisions and actions that have taken by both vessels.

If an autonomous vessel fails to satisfy the expected performance standards, then the recommendations on ship system improvements should be forwarded to the respective manufacture. Therefore, adequate modifications not only in navigation and automation systems but also a ship intelligence and decision support systems can be introduced (see Figure 5). It is also recommended to implement the following testing levels in the proposed platform to evaluate the behavior in autonomous vessels under various ship encounter situations:

- Testing level 1: Both autonomous and target vessels are simulated by software programs.

- Testing level 2: Target vessels are simulated by a software program and the autonomous ship is represented by a full-scale/model-scale vessel. The autonomous vessel is navigating in approved waters.

- Testing level 3: Both autonomous and target vessels are represented by fullscale/model-scale vessels. That are navigating in open sea.

It is expected that testing systems will initially evaluate vessel behavior under testing level 1. Both autonomous and target vessels can be considered as agent based systems in this situation, where appropriate mathematical models to simulate realistic ship behavior should be introduced. The respective sea trial data that are collected from ocean going vessels can be used to develop such mathematical models for ocean 
going vessels [27]. In general, such a mathematical model of ship maneuvering should consist of the following basic vectors: ship course-speed vector, heading vector and turning rates. That can also be estimated from time-series data sets, i.e. AIS data, and such data sets can also influence on the respective performance standards during their development phase. In addition, various weather conditions can also be introduced to create distinct navigation situations.

The lessons learned from testing level 1 should be transferred into testing level 2 that represents realistic ocean-going conditions. The implementation of testing level 3 can be a challenge due to the difficulties in creating vessel collision situations in open sea, intentionally (i.e. due to maneuverability difficulties of ocean going vessels). Therefore, the first and second testing levels can be considered as possible situations that can be implemented under the testing system. The outcomes of the two vessel encounter situations under these testing levels can be accumulated towards multivessel encounter situations, as mentioned previously.

These evaluation procedures of ship behavior, i.e. ship intelligence, in these testing systems should be developed under realistic ocean-going conditions. That can include all distinct navigation situations and ship encounter situations, i.e. varying environmental conditions, and random ship system and possible sensor failures, etc.. However, the main objective in such testing systems is to evaluate the vessel behavior of autonomous and target vessels rather than the reliability of hardware and software systems. Even though hardware and software system failures can eventually influence on ship intelligence and decision support layers of future vessels, a considerable number 
of tools and techniques to regulate such failures have been developed by the respective industries. In general, both hardware and software development processes are often guided by system (i.e. the $\mathrm{V}$ model) [28] and agile software development approaches [29] by the respective manufactures. It is expected that future vessels will have highly reliable hardware and software systems due to the technology maturity. Hence, adequate research studies should focus towards ship intelligence and decision support facilities of autonomous vessels, rather than individual system and component failures.

\section{CONCLUSIONS}

A structured framework to support the navigation considerations of autonomous vessels is discussed in this paper and that consists of various technologies to achieve the required integrity level of ocean autonomy. Since decision-making facilities in autonomous vessels will play an important role under ocean autonomy, the same technologies should consist of adequate system intelligence, so called ship intelligence. Each onboard application in autonomous ships may require a localized decision-making module, therefore a distributed intelligence type strategy as agent-based systems should be considered throughout these vessels. The main core of such an agent consists of deep learning type mathematical frameworks, i.e. ship intelligence, to mimic helmsman actions in ship navigation. A considerable amount of research and development activities will be required to achieve the required integrity level of ship intelligence, i.e. within deep learning type mathematical frameworks of autonomous vessels. One should note that various information visualization approaches in the bridge to enhance the learning process of ship intelligence should also be investigated. 
Furthermore, an additional decision supporting layer should also be available to facilitate distinct navigation situations in autonomous ships. It is expected that the combination of the ship intelligence and decision support layer can create adequate behavior to facilitate each distinct navigation situation.

Collision avoidance under situation awareness of future vessels is considered as such a distinct navigation situation in this study. Therefore, the required technologies, as a part of the decision support layer, to guide ship intelligence to avoid vessel collision situations in relation to the COLREGs are discussed. Furthermore, the respective evaluation steps of ship intelligence with the decision support facilities of collision avoidance under situation awareness with distinct navigation situations are also discussed. That should be done by a testing system of ship behavior, i.e. ship intelligence, and the outcome should be compared with the applicable limits of liability, terms, expectations and conditions in vessel navigation.

The required technologies to implement and evaluate the system intelligence in transport systems are still in a preliminary stage and the required knowledge yet to be created. The same knowledge should be shared among research communities to improve the navigation safety in relation to transport systems. Autonomous system developers will play a crucial role in developing and sharing such knowledge in system intelligence and decision support facilities and that may push machine learning into a more regulated industry. However, the human interactions and their outcome under system intelligence, i.e. artificial intelligence, should also be investigated by the research 
community. One should note that autonomous ships will also face the similar challenges under ocean autonomy.

The same outcome can be used to develop appropriate performance standards to evaluate vessel behavior as an agent based system by considering various encounter situations of future vessels. It is also noted that the respective navigation rules and regulations may have possible regulatory failure situations under the testing system, therefore adequate measures to overcome such challenges should be also considered. Finally, the required technologies, their capabilities and applicability (i.e. including regulatory failures), and the implementation and evaluation challenges with possible solutions for collision avoidance under situation awareness of autonomous vessels are extensively summarized in this study as the main contribution.

\section{ACKNOWLEDGMENT}

An initial version of this paper is presented at the 37th International Conference on Ocean, Offshore and Arctic Engineering (OMAE 2018), Madrid, Spain, June, 2018 (OMAE2018-77672).

\section{REFERENCES}

[1] Li, T. H. S., Lee, M. H., Lin, C. W., Liou, G. H. and Chen, W. C., 2016, "Design of Autonomous and Manual Driving System for 4WIS4WID Vehicle," in IEEE Access, 4, pp. 2256-2271.

[2] Dokic, J., Müller, B., and Meyer, G., 2015, European Roadmap Smart Systems for Automated Driving, European Technology Platform on Smart Systems Integration (EPoSS).

[3] Rahman, A. A., Hamid, U. Z. A., Rahman, T. A. C., 2017, "Emerging Technologies with Disruptive Effects: A Review" PERINTIS eJournal. 7 (2). 
[4] Yara Birkeland, 'The first ever zero emission, autonomous ship,' URL: https://www.yara.com/knowledge-grows/game-changer-for-the-environment/, online: 2019.

[4] Perera, L. P., 2017, "Industrial IoT to Predictive Analytics: A Reverse Engineering Approach from Shipping," In Proceedings of the 3rd Norwegian Big Data Symposium (NOBIDS), Trondheim, Norway.

[5] Perera, L. P., Ferrari, V., Santos, F. P., Hinostroza, M. A., and Guedes Soares, C., 2015, "Experimental Evaluations on Ship Autonomous Navigation \& Collision Avoidance by Intelligent Guidance," IEEE Journal of Oceanic Engineering, 40(2), pp. 374-387.

[6] Rolls-Royce, 2016, "Remote and Autonomous ship: the next step," AAWA position paper.

[7] Panait, L. and Luke, S., 2005, "Cooperative Multi-Agent Learning: The State of the Art", Autonomous Agents and Multi-Agent Systems, 11(3), pp. 387-434

[8] Wooldridge, M., and Jennings, N., 1995, "Intelligent agents: Theory and practice," The Knowledge Engineering Review, 10(2), pp. 115-152.

[9] Rolls-Royce, 2016, Ship intelligence transforming future marine operations, Norway.

[10] Giusti, A., Guzzi, J., Ciresan, D.C., He, F., Rodriguez, J.P., Fontana, F., Faessler, M., Forster, C., Schmidhuber, J., Di Caro, G., Scaramuzza, D., and Gambardella, L.M., 2016, "A Machine Learning Approach to Visual Perception of Forest Trails for Mobile Robots, " IEEE Robotics and Automation Letters (RA-L), pages $661-667$.

[11] Perera, L.P., and Guedes Soares, C., 2015, "Collision Risk Detection and Quantification in Ship Navigation with Integrated Bridge Systems," Journal of Ocean Engineering, 109, pp. 344-354.

[12] Liu, S., Tang, J., Zhang, Z., and Gaudiot, J. L., 2017, "Computer Architectures for Autonomous Driving," in Computer, 50(8), pp. 18-25.

[13] Collobert, R., and Weston, J., 2008, "A Unified Architecture for Natural Language Processing: Deep Neural Networks with Multitask Learning". Proceedings of the 25th International Conference on Machine Learning. ICML '08. New York, USA: ACM: 160167.

[14] Perera, L.P., and Guedes Soares, C., 2017, "Weather Routing and Safe Ship Handling in the Future of Shipping," Journal of Ocean Engineering, 130, pp. 684-695. 
[15] Bresson, G., Alsayed, Z., Yu, L., and Glaser, S., 2017, "Simultaneous Localization and Mapping: A Survey of Current Trends in Autonomous Driving," in IEEE Transactions on Intelligent Vehicles, 2(3), pp. 194-220.

[16] Perera, L. P., Rodrigues, J. M., Pascoal, R. and C. Guedes Soares, C., 2012, "Development of an onboard decision support system for ship navigation under rough weather conditions," Sustainable Maritime Transportation and Exploitation of Sea Resources, E. Rizzuto \& C. Guedes Soares (Eds.), Taylor \& Francis Group, London, UK, pp. 837-844.

[17] Szegedy, C., Toshev, A., and Erhan, D., 2013, "Deep neural networks for object detection". Advances in Neural Information Processing Systems.

[18] The Munin Project, 2018, [online] Available: http://www.unmannedship.org/munin/wp.

[19] Burmeister, H.-C., Bruhn, W., Rødseth, $\varnothing$. J., Porathe, T., 2014, “Autonomous Unmanned Merchant Vessel and its Contribution towards the e-Navigation Implementation: The MUNIN Perspective," International Journal of e-Navigation and Maritime Economy, 1, pp. 1-13.

[20] IMO, 1972, "Convention on the International Regulations for Preventing Collisions at Sea (COLREGs)," [Online]. Available: http://www.imo.org/conventions

[21] Rothblum, A.M., Wheal, D., Withington, S., Shappell, S. A., Wiegmann, D. A., Boehm, W., and Chaderjian, M., 2002, "Key to successful incident inquiry," in Proc. 2nd Int. Workshop Human Factors Offshore Oper., pp. 1-6.

[22] Perera, L.P., Carvalho, J.P., and Guedes Soares, C., 2012, "Intelligent ocean navigation \& Fuzzy-Bayesian decision-action formulation," IEEE Journal of Oceanic Engineering, 37(2), pp. 204-219.

[23] Perera, L.P., Carvalho, J.P., and Guedes Soares, C., 2014, "Solutions to the Failures and Limitations of Mamdani Fuzzy Inference in Ship Navigation," IEEE Transactions on Vehicular Technology, 63(4), pp. 1539-1554.

[24] Perera, L.P. and Batalden, B.M., 2019 "Possible COLREGs Failures under Digital Helmsman of Autonomous Ships," In Proceedings of the MTS/IEEE OCEANS 19, Marseille , France.

[25] IMO, 2002, 'Standards for Ship Manoeuvrability,' Resolution MSC.137 (76).

[26] IMO, 2002, 'Explanatory Notes to the Standards for Ship Manoeuvrability,' MSC/Circ.1053. 
[27] International standard ISO 15016 and ISO 19019, 2002, "Ship and marine technology - Guidelines for the assessment of speed and power performance by analysis of speed trial data", First edition.

[28] Forsberg, K. and Mooz, H., 1992, "The Relationship of System Engineering to the Project Cycle", Engineering Management Journal, 4(3), pp. 36-43.

[29] Abrahamson, P., Salo, O., Ronkainen, J., and Warsta, J., 2002. Agile software development methods: Review and analysis (Technical report). VTT. 478. 
Journal of Offshore Mechanics and Arctic Engineering

\section{Figure Captions List}

Fig. 1 A structured technology framework for autonomous ship navigation

Fig. 2 Ship encounter situation 1

Fig. 3 Ship encounter situation 2

Fig. $4 \quad$ Ship encounter situation 3

Fig. 5 Testing system for evaluating autonomous vessel behavior 


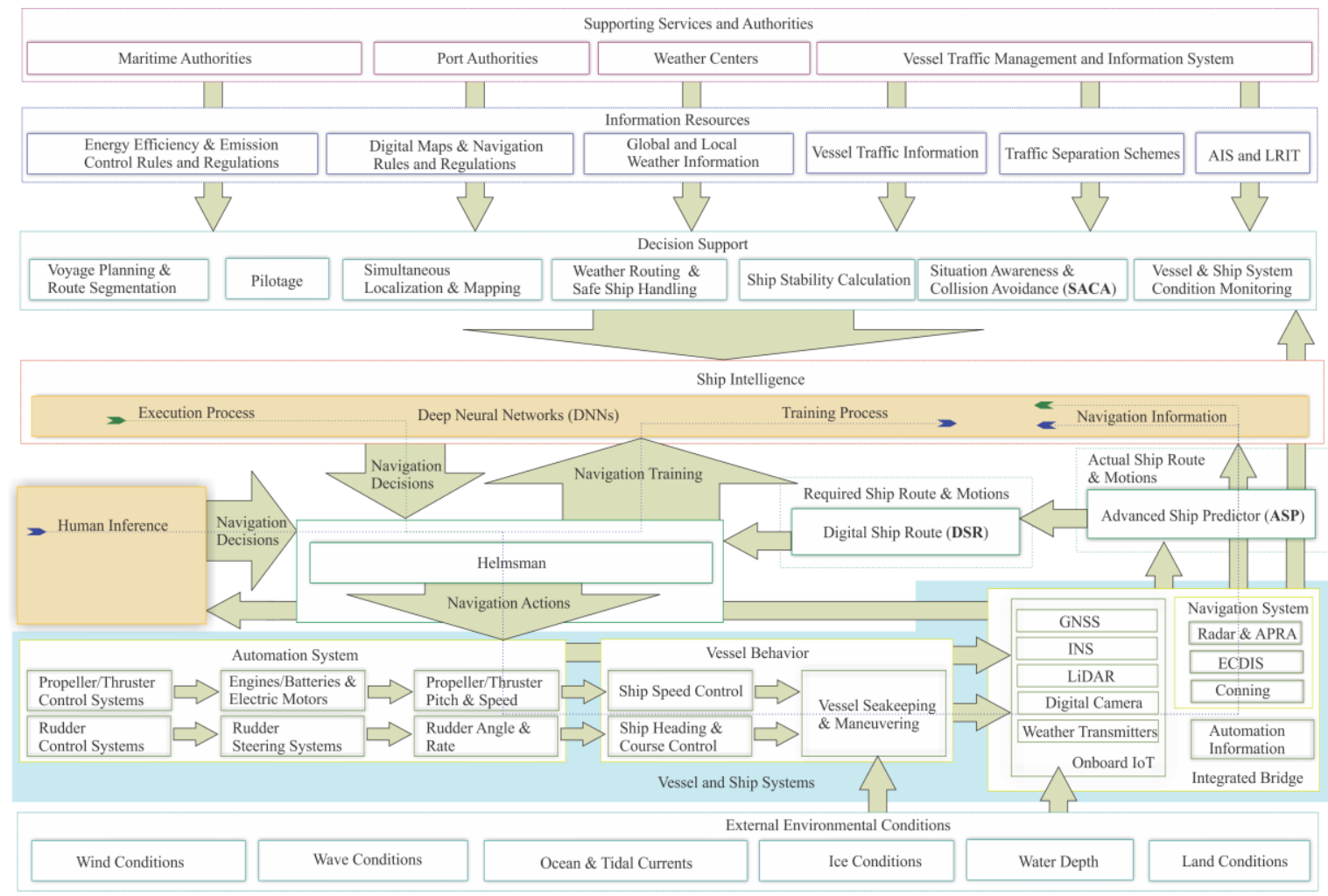

Fig. 1 A structured technology framework for autonomous ship navigation 


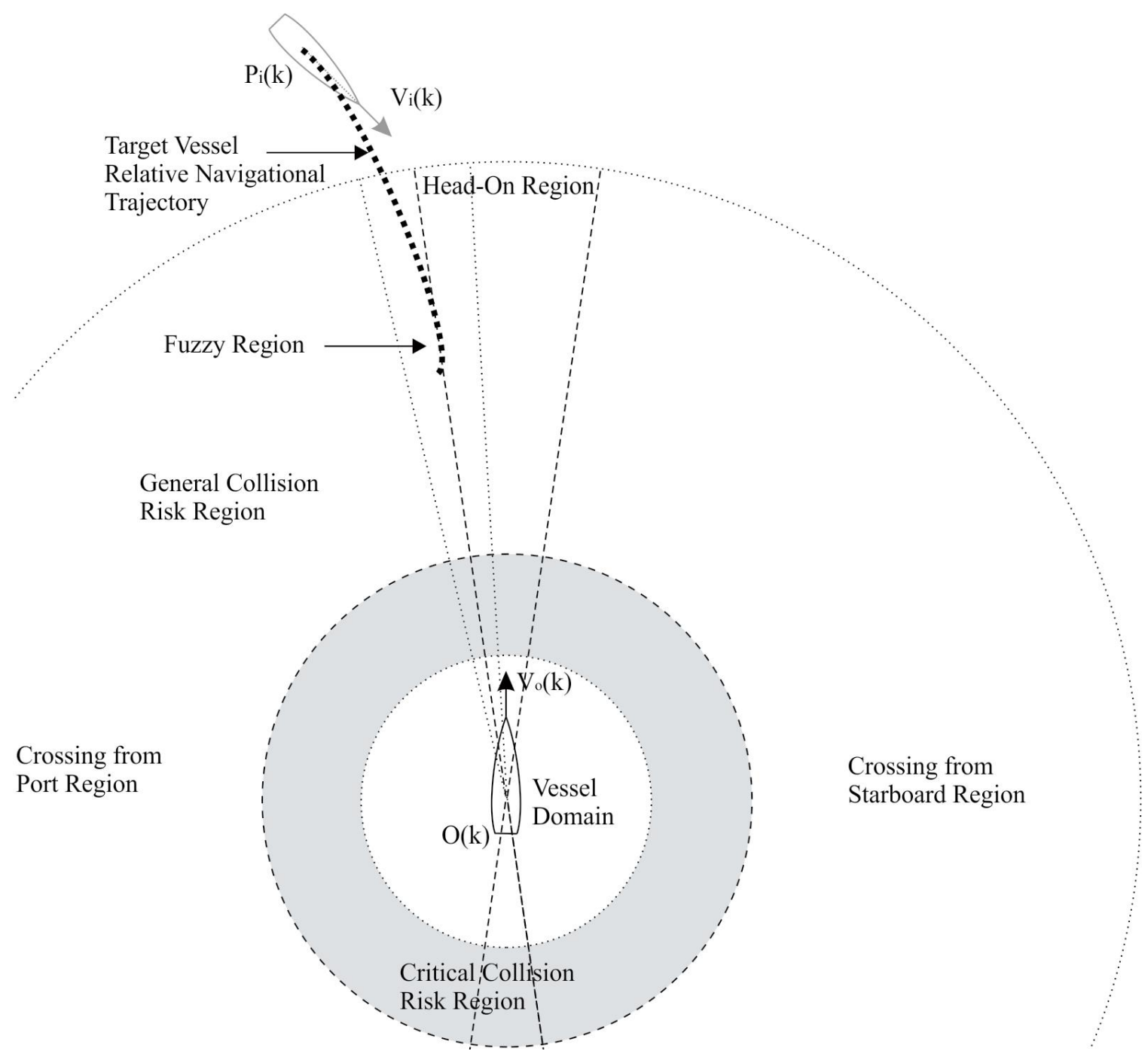

Fig 2: Ship encounter situation 1 


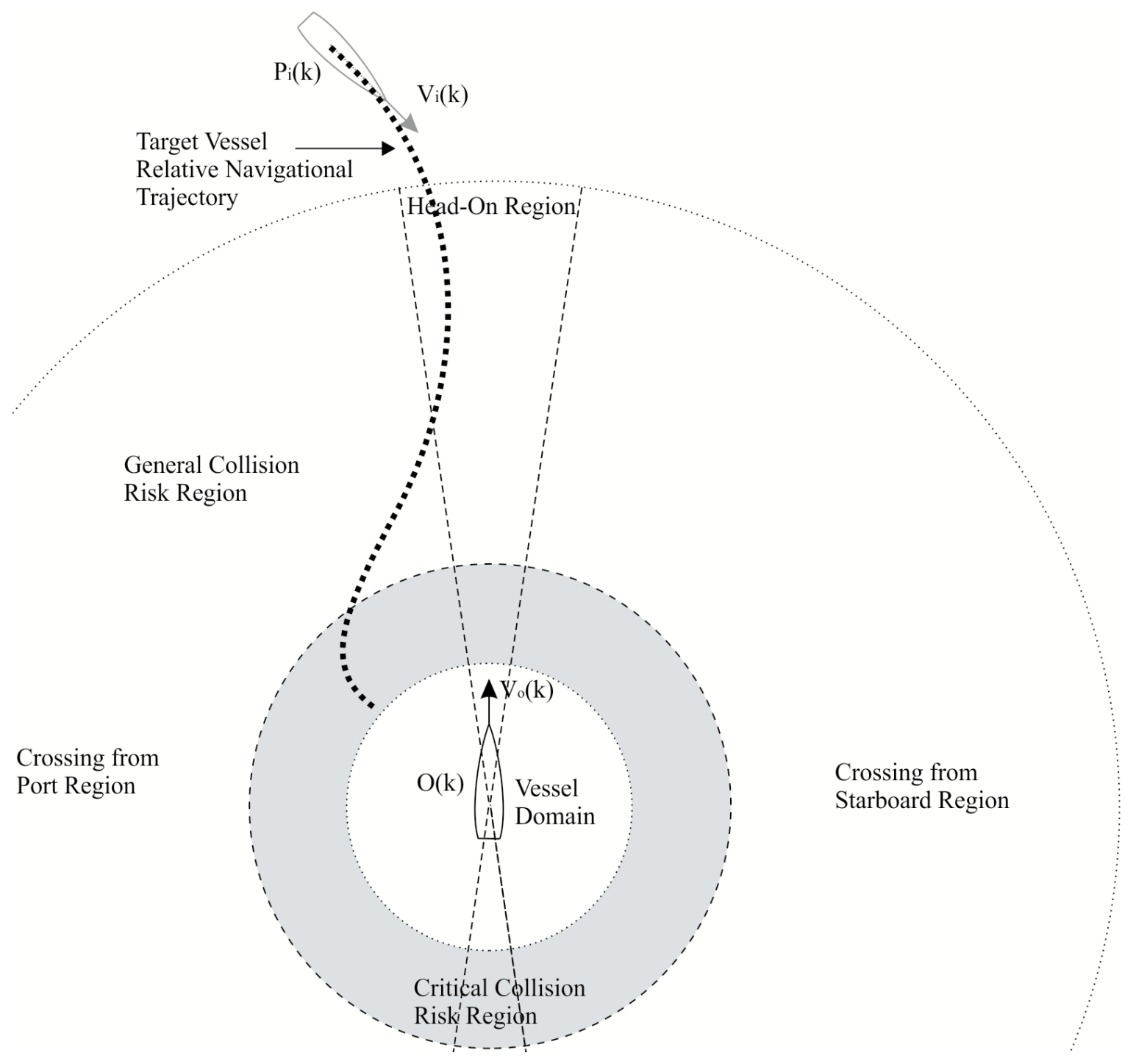

Fig 3: Ship encounter situation 2 


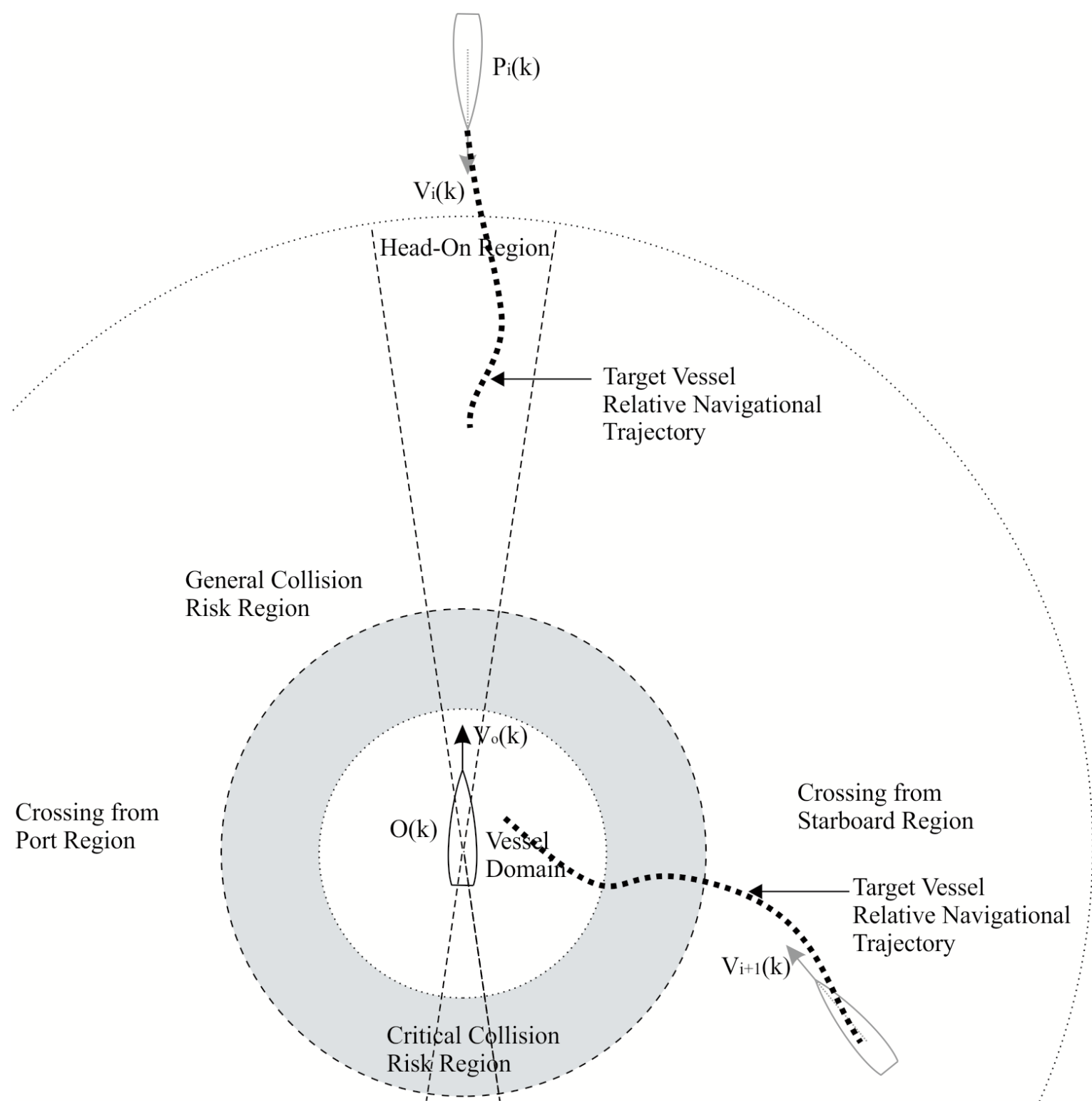

Fig 4: Ship encounter situation 3 


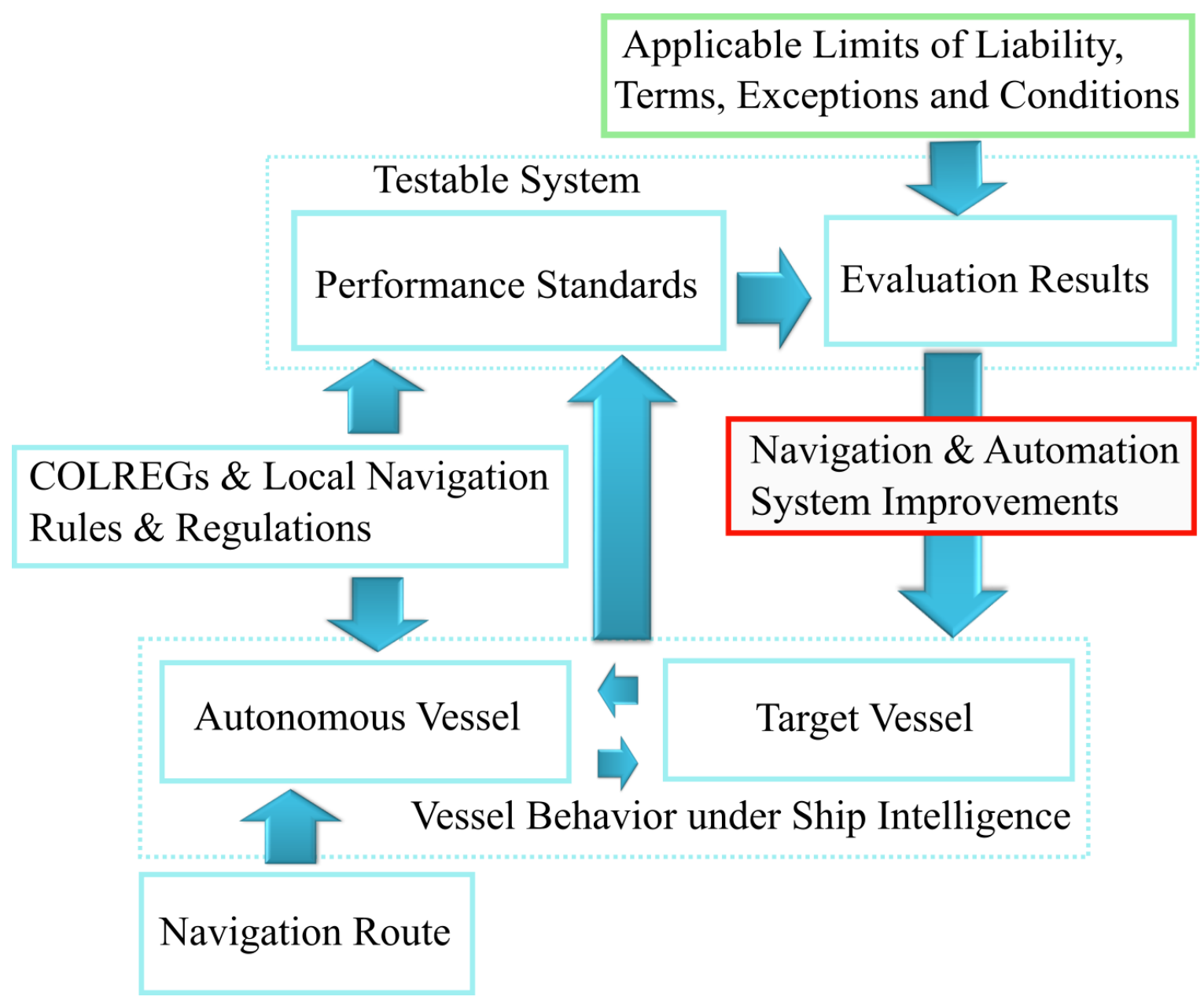

Fig 5: Testing system for evaluating autonomous vessel behavior 